If we let $\omega$ be any of the possible solutions of

$$
x=\beta(\omega), \quad g(0) \leqq \omega \leqq g(1),
$$

we may write (4) in the form

$$
F(\omega)=M(\omega)+\lambda \int_{g(0)}^{g(1)} k(\omega, s) F(s) d s,
$$

where $F(\omega)=f(\beta(\omega)), \quad M(\omega)=m(\beta(\omega)), k(\omega, s)=K(\beta(\omega), \beta(s))$. We thus have our main result:

Theorem 3. When $G(x, y)$ is absolutely continuous $g(y)$ the Fredholm-Stieltjes integral equation (1) is reducible to an ordinary Fredholm integral equation.

4. Mixed linear equations. The mixed equation*

$$
f(x)=m(x)+\sum_{i=1}^{m} \lambda K^{(i)}(x) f\left(s_{i}\right)+\lambda \int_{0}^{1} K(x, s) f(s) d s
$$

can easily be put into the form

$$
f(x)=m(x)+\lambda \int_{0}^{1} R(x, s) f(s) d g(s) .
$$

Thus from Theorem 3 we see that equation (5) is reducible to a Fredholm integral equation.

Duke UnIVERsity

\title{
A THEOREM ON QUADRATIC FORMS $\dagger$
}

\section{WILLIAM T. REID}

In this note the following result is proved:

ThEOREM. Suppose $A[x] \equiv a_{\alpha \beta} x_{\alpha} x_{\beta}, \ddagger B[x] \equiv b_{\alpha \beta} x_{\alpha} x_{\beta}$ are real quadratic forms in $\left(x_{\alpha}\right),(\alpha=1, \cdots, n)$, and that $A[x]>0$ for all real $\left(x_{\alpha}\right) \neq\left(0_{\alpha}\right)$ satisfying $B[x]=0$. Then there exists a real constant $\lambda_{0}$ such that $A[x]-\lambda_{0} B[x]$ is a positive definite quadratic form.

This theorem is of use in considering the Clebsch condition for multiple integrals in the calculus of variations. A. A. Albert $\$$ has given

* W. A. Hurwitz, Mixed linear integral equations of the first order, Transactions of this Society, vol. 16 (1915), pp. 121-133.

$\dagger$ Presented to the Society, December 30, 1937.

$\ddagger$ The tensor analysis summation convention is used throughout.

$\S$ This Bulletin, vol. 44 (1938), pp. 250-253. 
an algebraic proof of this result. The following proof is more analytic in character than that of Albert and is of interest because in the proof itself we obtain directly, in terms of the roots of the characteristic equation (1) below, the interval in which the value $\lambda_{0}$ of the theorem may be chosen.

Suppose that the matrix $\left\|b_{\alpha \beta}\right\|$ has rank $n-r,(0 \leqq r<n)$, and let $u_{\alpha}=u_{\alpha \kappa},(\kappa=1, \cdots, r)$, be linearly independent solutions of the equations $b_{\alpha \beta} u_{\beta}=0,(\alpha=1, \cdots, n)$.

LEMMa 1. The characteristic equation

$$
D(\lambda) \equiv\left|a_{\alpha \beta}-\lambda b_{\alpha \beta}\right|=0
$$

is a polynomial of degree $n-r$ in $\lambda$.

Let $u_{\alpha r+s},(s=1, \cdots, n-r)$, be sets linearly independent of the above defined $u_{\alpha \kappa}$ and such that the determinant $\left|u_{\alpha \beta}\right|$, $(\alpha, \beta=1, \cdots, n)$, is different from zero. Clearly the degree of $D(\lambda)$ is equal to that of the polynomial $D_{1}(\lambda)=\left|\left(a_{\alpha \gamma}-\lambda b_{\alpha \gamma}\right) u_{\gamma \beta}\right|$. Since the first $r$ columns of $D_{1}(\lambda)$ are independent of $\lambda$, we obviously have $d^{k} D_{1} / d \lambda^{k} \equiv 0$ if $k>n-r$. Also, the $(n-r)$ th derivative is equal to $(n-r) !\left|a_{\alpha \gamma} u_{\gamma \kappa}-b_{\alpha \gamma} u_{\gamma+s+s}\right|,(\kappa=1, \cdots, r ; s=1, \cdots, n-r)$; and if its value is zero, there are constants $\left(c_{\alpha}\right) \neq\left(0_{\alpha}\right)$ such that $u_{\alpha}^{0}=u_{\alpha \kappa} c_{\kappa}, u_{\alpha}^{\prime}=u_{\alpha r+s} c_{r+s}$ satisfy the equations $a_{\alpha \beta} u_{\beta}^{0}=b_{\alpha \beta} u_{\beta}^{\prime}$. By use of these equations together with $u_{\alpha}^{0} b_{\alpha \beta}=0_{\beta}$ we have $A\left[u^{0}\right]=0$; hence $\left(u_{\alpha}^{0}\right)=\left(0_{\alpha}\right)$, by the hypothesis of the theorem, and consequently $\left(c_{\kappa}\right)=\left(0_{\kappa}\right)$. This implies in turn that $b_{\alpha \beta} u_{\beta}^{\prime}=0_{\alpha} ;$ and since $\left(u_{\alpha}^{\prime}\right) \neq\left(0_{\alpha}\right)$ and is independent of the sets $u_{\alpha \kappa},(\kappa=1, \cdots, r)$, we have a contradiction to the assumption that $\left\|b_{\alpha \beta}\right\|$ is of rank $n-r$. The above lemma is therefore proved.

Corresponding to a root of (1) the number of linearly independent solutions of the associated equations

$$
\left(a_{\alpha \beta}-\lambda b_{\alpha \beta}\right) y_{\beta}=0_{\alpha}, \quad \alpha, \beta=1, \cdots, n,
$$

is termed the index of $\lambda$ as a root of (1). For sets $x_{\alpha}^{\prime} \equiv\left(x_{\alpha}^{\prime}\right), x_{\alpha}^{\prime \prime} \equiv\left(x_{\alpha}^{\prime \prime}\right)$ we denote by $A\left[x^{\prime} ; x^{\prime \prime}\right], B\left[x^{\prime} ; x^{\prime \prime}\right]$ the bilinear forms $a_{\alpha \beta} x_{\alpha}^{\prime} x_{\beta}^{\prime \prime}$, $b_{\alpha \beta} x_{\alpha}{ }^{\prime} x_{\beta}{ }^{\prime \prime}$. The first of the following lemmas is immediate.

LEMma 2. If $\lambda^{\prime}, \lambda^{\prime \prime}$ are distinct zeros of $D(\lambda)$, and $y_{\alpha}^{\prime}, y_{\alpha}^{\prime \prime}$ are solutions of the corresponding equations (2), then $B\left[y^{\prime} ; y^{\prime \prime}\right]=0=A\left[y^{\prime} ; y^{\prime \prime}\right]$.

Lemмa 3. The zeros of $D(\lambda)$ are all real, and the corresponding solutions of (2) may be chosen real. 
For suppose $\lambda^{\prime}=\lambda_{1}+i \lambda_{2},\left(\lambda_{2} \neq 0\right)$, is a zero of $D(\lambda)$, and $y_{\alpha}^{\prime}=y_{\alpha 1}+i y_{\alpha 2}$ is a corresponding solution of (2). Then the conjugate values $\bar{y}_{\alpha}$ satisfy (2) for $\lambda=\bar{\lambda}^{\prime}$. By Lemma 2 we have $0=A[y ; \bar{y}]=A\left[y_{1}\right]+A\left[y_{2}\right]$, $0=B[y ; \bar{y}]=B\left[y_{1}\right]+B\left[y_{2}\right]$. Now if either $B\left[y_{1}\right]$ or $B\left[y_{2}\right]$ is zero, so also is the other, and, in view of the hypothesis of the theorem, we have a contradiction. On the other hand, if $B\left[y_{2}\right] \neq 0$, let $c$ be a value opposite in sign to $\lambda_{2} / B\left[y_{1}\right]$ and such that $0=B\left[y_{1}+c y_{2}\right]$ $=B\left[y_{1}\right]+2 c B\left[y_{1} ; y_{2}\right]+c^{2} B\left[y_{2}\right]=\left(1-c^{2}\right) B\left[y_{1}\right]+2 c B\left[y_{1} ; y_{2}\right]$. Using the relations satisfied by $y_{\alpha 1}, y_{\alpha 2}$ one obtains, by direct calculation, $A\left[y_{1}+c y_{2}\right]=\left(2 c \lambda_{2} / B\left[y_{1}\right]\right)\left(\left\{B\left[y_{1}\right]\right\}^{2}+\left\{B\left[y_{1} ; y_{2}\right]\right\}^{2}\right)<0$, which is a contradiction. Hence there are no complex zeros of $D(\lambda)$, and the solutions of equations (2) may be chosen real.

Lemma 4. If $\lambda=\lambda^{\prime}$ is a zero of $D(\lambda)$, then its index is equal to its multiplicity.

Suppose the index of $\lambda^{\prime}$ is equal to $k$, and denote by $y_{\alpha}=y_{\alpha j}$, $(j=1, \cdots, k)$, corresponding linearly independent solutions of (2). Now choose $y_{\alpha h},(h=k+1, \cdots, n)$, such that the determinant $\left|y_{\alpha \beta}\right|$, $(\alpha, \beta=1, \cdots, n)$, is different from zero. Clearly the multiplicity of $\lambda^{\prime}$ as a zero of $D(\lambda)$ is equal to its multiplicity as zero of

$$
\Delta(\lambda) \equiv\left|\left(a_{\alpha \gamma}-\lambda b_{\alpha \gamma}\right) y_{\gamma \beta}\right| \text {. }
$$

Since the first $k$ columns of $\Delta(\lambda)$ are zero for $\lambda=\lambda^{\prime}$, the multiplicity of $\lambda^{\prime}$ as a zero of $\Delta(\lambda)$ is not less than $k$. Moreover,

$$
\begin{array}{r}
d^{k} \Delta /\left.d \lambda^{k}\right|_{\lambda=\lambda^{\prime}}=k !\left|-b_{\alpha \beta} y_{\beta j}\left(a_{\alpha \beta}-\lambda^{\prime} b_{\alpha \beta}\right) y_{\beta h}\right|, \\
j=1, \cdots, k ; h=k+1, \cdots, n .
\end{array}
$$

Now if this derivative is zero, there are constants $\left(c_{\alpha}\right) \neq\left(0_{\alpha}\right)$ such that the set $y_{\alpha}^{0}=y_{\alpha j} c_{j}, y_{\alpha}^{\prime}=y_{\alpha h} c_{h}$ satisfies

$$
\left(a_{\alpha \beta}-\lambda^{\prime} b_{\alpha \beta}\right) y_{\beta}^{\prime}=b_{\alpha \beta} y_{\beta}^{0} \text {. }
$$

Since $\left(y_{\alpha}^{0}\right)$ satisfies (2) for $\lambda=\lambda^{\prime}$ we find from (4) that $B\left[y^{0}\right]=0$, and consequently, using again equations (2), that $A\left[y^{0}\right]=0$. In view of the hypothesis of the theorem it then follows that $\left(y_{\alpha}^{0}\right)=\left(0_{\alpha}\right)$ and $\left(c_{j}\right)=\left(0_{j}\right)$. But from equations (4) we see that $\left(y_{\alpha}^{\prime}\right)$ is then a solution of (2) for $\lambda=\lambda^{\prime}$; and, since this set is independent of the $k$ sets $y_{\alpha j}$, we have a contradiction to the assumption that $k$ is the index of $\lambda^{\prime}$. Hence the expression (3) is not zero, and the multiplicity of $\lambda^{\prime}$ as a zero of $D(\lambda)$ is also $k$.

Now let $\lambda_{1} \leqq \lambda_{2} \leqq \ldots . \lambda_{n-r}$ denote the zeros of $D(\lambda)$ each repeated a number of times equal to its index, and let $y_{\alpha}=y_{\alpha s},(s=1, \cdots, n-r)$, be corresponding linearly independent real solutions of equations (2). 
LEMMA 5. The solutions $y_{\alpha s}$ are such that $B\left[y_{s}\right] \neq 0,(s=1, \cdots, n-r)$; moreover, these solutions may be so chosen that $B\left[y_{s} ; y_{t}\right]=0$ if $s \neq t$.

The first part of the lemma follows readily from the hypothesis of the theorem, since $A\left[y_{s}\right]=\lambda_{s} B\left[y_{s}\right],(s=1, \cdots, n-r)$. If $\lambda_{s} \neq \lambda_{t}$, the second part of the lemma is true by Lemma 2. If $\lambda_{s}=\lambda_{t}$, the corresponding solutions may be so orthogonalized in view of the relation $B\left[y_{s}\right] \neq 0$.

COROLLARY. The determinant $\left|u_{\alpha \kappa} y_{\alpha s}\right|$ is different from zero.

LEMMA 6. If for a value s we have $B\left[y_{s}\right]>0$, then for each $t$ such that $\lambda_{t} \geqq \lambda_{s}$ we also have $B\left[y_{t}\right]>0$.

For suppose $\lambda_{t} \geqq \lambda_{s}$ and $B\left[y_{t}\right]<0$. If $c^{2}=-B\left[y_{t}\right] / B\left[y_{s}\right]$, then by the above lemma we have $\left(y_{\alpha t}+c y_{\alpha s}\right) \neq\left(0_{\alpha}\right)$. Moreover, $B\left[y_{t}+c y_{s}\right]$ $=B\left[y_{t}\right]+c^{2} B\left[y_{s}\right]=0, A\left[y_{t}+c y_{s}\right]=A\left[y_{t}\right]+c^{2} A\left[y_{s}\right]=B\left[y_{t}\right]\left(\lambda_{t}-\lambda_{s}\right) \leqq 0$, which is impossible in view of the hypothesis of the theorem.

Now let $\Lambda$ denote the interval of the $\lambda$ axis defined by $\lambda<\lambda_{1}$ if $B\left[y_{1}\right]>0, \lambda>\lambda_{n-r}$ if $B\left[y_{n-r}\right]<0, \lambda_{g}<\lambda<\lambda_{g+1}$ if $B\left[y_{g}\right]<0, B\left[y_{g+1}\right]>0$. The interval $\Lambda$ is uniquely determined in view of Lemma 6 . We shall now show that $A[x]-\lambda_{0} B[x]$ is a positive definite quadratic form for $\lambda_{0}$ on $\Lambda$. If $\left(x_{\alpha}\right) \neq\left(0_{\alpha}\right)$, it follows from the corollary to Lemma 5 that there are unique values $\left(c_{\kappa}, d_{s}\right) \neq\left(0_{\kappa}, 0_{s}\right)$ such that $x_{\alpha}=u_{\alpha \kappa} c_{\kappa}$ $+y_{\alpha s} d_{s}$. Moreover, since $u_{\alpha \kappa} b_{\alpha \beta}=0$, we have $A\left[u_{k} ; y_{s}\right]=0, \quad(\kappa=1$, $\cdots, r ; s=1, \cdots, n-r)$, and $A\left[u_{\kappa}, u_{\nu}\right] c_{k} c_{\nu} \geqq 0$, with the inequality sign holding unless $\left(c_{k}\right)=\left(0_{k}\right)$. Now if $\lambda_{0}$ is on the above defined interval $\Lambda$, each of the terms $\left(\lambda_{s}-\lambda_{0}\right) B\left[y_{s}\right]$ is positive. From the relations

$$
\begin{aligned}
A[x]-\lambda_{0} B[x] & =A\left[u_{k} ; u_{\nu}\right] c_{\kappa} c_{\nu}+\left(A\left[y_{s} ; y_{t}\right]-\lambda_{0} B\left[y_{s} ; y_{t}\right]\right) d_{s} d_{t} \\
& =A\left[u_{k} ; u_{\nu}\right] c_{\kappa} c_{\nu}+\sum_{s=1}^{n-r}\left(\lambda_{s}-\lambda_{0}\right) B\left[y_{s}\right] d_{s}^{2}
\end{aligned}
$$

we therefore conclude that $A[x]-\lambda_{0} B[x]$ is positive definite for $\lambda$ on $\Lambda$. In conclusion it is to be noted that if $\lambda_{0}$ is not on this interval, the form $A[x]-\lambda_{0} B[x]$ is negative or zero for at least one of the sets $\left(x_{\alpha}\right)=\left(y_{\alpha s}\right),(s=1, \cdots, n-r)$.

UNiversity of Chicago 